\title{
Experimental Acanthamoeba keratitis: I. Preliminary findings
}

\author{
D F P Larkin, D L Easty
}

\begin{abstract}
Experimental Acanthamoeba keratitis was induced in Wistar rats by intrastromal inoculation of Acanthamoeba polyphaga cysts. Keratitis developed in all corneas injected and was characterised by a diffuse granular opacity. The animals were examined for 70 days and a gradual reduction of keratitis was observed. Pathological sections showed liquefactive stromal necrosis and Acanthamoeba localised in deep stroma persisting to $\mathbf{7 0}$ days. A brisk inflammatory infiltrate of neutrophils and macrophages was seen in the early days of infection. Severity of keratitis was found to correlate with inflammatory activity in pathological sections. The uses of an animal model of Acanthamoeba keratitis are described.
\end{abstract}

Acanthamoeba is a genus of free-living protozoon with a ubiquitous distribution in nature. It has a life cycle of active trophozoite and dormant cyst stages. First isolated and described in $1913,{ }^{1}$ it was not considered to have pathogenic potential until reported to cause fatal meningoencephalitis in animal inoculation experiments in $1959 .{ }^{2}$ Some years later, when suspected amoebic encephalitis was reported in humans, the similarity to Acanthamoeba infection induced experimentally was recognised and suggested the aetiological role of Acanthamoeba. The pathological features of human encephalitis are so similar to those of experimental Acanthamoeba encephalitis in mice that the use of animal models for studying other forms of Acanthamoeba infection is clearly indicated. ${ }^{3}$

After initial reports ${ }^{4}$ Acanthamoeba infection of the cornea remained a rare disease until an association with contact lens wear was first recognised. ${ }^{6}$ Reports of cases have exponentially increased since that time, and a recent analysis identified $85 \%$ of affected patients as contact lens wearers. ${ }^{7}$ Acanthamoeba keratitis typically has an indolent but progressive course, with intense discomfort and stromal infiltration. Therapy of Acanthamoeba keratitis is difficult in all cases, with frequent failures of medical and surgical treatment and loss of the eye in some of these.

Even though the keratitis has become more common than the encephalitis, few laboratory in-vivo studies have been reported. Rabbit models have been developed, first by Font and colleagues. ${ }^{89}$ After subconjunctival administration of corticosteroid for four days the direct intrastromal injection of $A$ polyphaga induced stromal keratitis and corneal ulceration. Some corneas were culture-positive at intervals, and encysted amoebae were observed in tissue on microscopy. A further rabbit model was reported by Lin and colleagues in which the effect of steroid administration was analysed. ${ }^{10}$ Badenoch et al reported a model in Porton rats in which induction of Acanthamoeba keratitis was dependent on coinoculation with bacteria. ${ }^{11}$

In this study the Wistar rat was used as the experimental animal. The initial objective of the study was to induce reproducible disease similar to the human disease, for use in evaluation of immunopathology and therapeutic agents. So that the specific immune response to Acanthamoeba could be studied coinoculation with bacteria was avoided.

\section{Materials and methods}

Animals. Male Wistar rats weighing approximately $125 \mathrm{~g}$ were used. The corneas were examined prior to inoculation to exclude abnormality (Fig 1A). There were 24 rats in the infected group and six in the control group. All procedures were performed on the right eye.

Amoeba. All studies were performed with the Shinh strain of $A$ polyphaga, a human corneal isolate (kindly provided by $\mathrm{Mr} \mathrm{S}$ Kilvington, Public Health Laboratory, Bath). This had been frozen in liquid nitrogen and passaged on a Vero cell monolayer three times. An axenic suspension of $1 \times 10^{6}$ amoebae $/ \mathrm{ml}$ ( $90 \%$ cysts) was prepared in Page's amoeba saline. ${ }^{12}$

Anaesthesia. Diazepam $0.25 \mathrm{mg}$ and fentanyl $0.01 \mathrm{mg} /$ fluanisone $0.3 \mathrm{mg}$ were given by intramuscular injection, and one drop of preservativefree benoxinate was applied to the right eye.

Inoculation technique. The procedure was performed under an operating microscope (OPMI 1 FC, Carl Zeiss Ltd). Initially a half thickness linear blade incision was made approximately $2 \mathrm{~mm}$ from the centre of the cornea. With a microlitre syringe (Hamilton Bonaduz AG) and a sterile $30 \mathrm{G}$ needle (BectonDickinson \& Co.), the needle was advanced from the incision through the lamellae of the stroma to the centre of the cornea. $1 \mu \mathrm{l}$ was injected into the stroma. Control animals received a mock inoculum of $1 \mu \mathrm{l}$ of Page's saline; infection animals received a suspension of $1 \times 10^{6}$ amoebae/ $\mathrm{ml}$ ( $90 \%$ cysts) in Page's amoeba saline.

Clinical evaluation Under fentanyl general anaesthesia the rats were examined with a slitlamp microscope on days 1, 3, 5 and 7 following inoculation and weekly therafter until 70 days. Examination was by retroillumination, resulting in transillumination of the albino iris and the cornea. The oblique slit beam was used to illuminate lesser degrees of opacity. A clinical grading system was used to describe corneal opacity:

Grade 3: opacity visible on retroillumination, obscuring iris vessel detail.

\author{
Correspondence to: \\ Mr D F P Larkin, Moorfield \\ Eye Hospital, City Road, \\ Accepted for publication \\ 29 March 1990 \\ Ophthalmology, Bristol \\ Eye Hospital, Bristol
}


Figure 1A Normal rat cornea with transillumination of albino iris seen on retroillumination.

Figure 1B Day 5: infected rat cornea showing diffuse granular central corneal opacity.
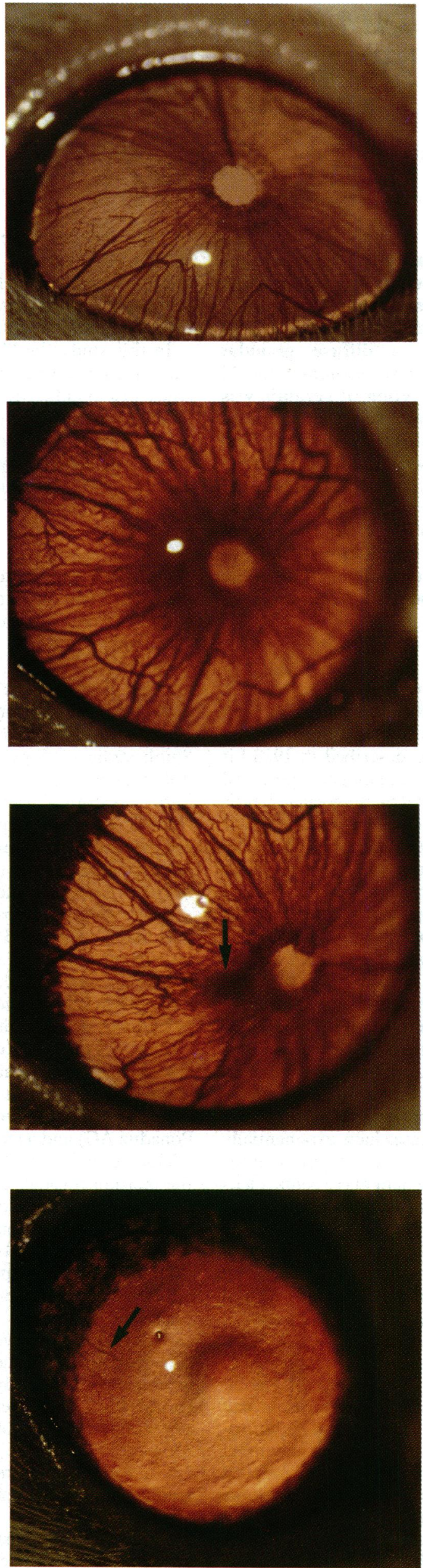

Grade 2: opacity visible on retroillumination, but not sufficient to obscure iris vessels.

Grade 1: opacity visible only by oblique slit beam.

Grade 0: normal.

A mean opacity grade was calculated for the infected corneas on each examination day. Photographs were taken with the operating microscope with retroillumination of the cornea, 160 ASA tungsten film (Kodak Ektachrome) and automatic exposure.

On days $1,3,7,14,21,28,49$, and 70 after inoculation two infected rats were killed by intraperitoneal injection of $60 \mathrm{mg}$ pentobarbitone. Two control rats were killed on days 1,3 , and 7.

Tissue processing. The eye was enucleated and the cornea dissected from the globe and hemisected. One half of the cornea was fixed for $24 \mathrm{~h}$ in neutral buffered formaldehyde, embedded in wax, and $6 \mu \mathrm{m}$ thickness sections cut with a microtome; the other half was either immediately frozen in liquid nitrogen or homogenised with a glass grinder for culture.

Preparation of specific antibody. Rabbit antiserum was prepared to the experimental strain of $A$ polyphaga according to the method described by Warhurst. ${ }^{13}$ Briefly, a $2.5 \mathrm{~kg} \mathrm{New}$ Zealand White rabbit was injected intramuscularly with $30 \mathrm{ml} A$ polyphaga/Freund's complete adjuvant and this was repeated at a three-week interval. This was followed by two $0.6 \mathrm{ml}$ intravenous injections of formalinised $A$ polyphaga cysts at four and five weeks. After venepuncture at six weeks the antiserum titre was measured and found to be $1 / 1024$. The serum was then affinity purified on a chromatography column using protein A-Sepharose (Sepharose CL-4B, Pharmacia, Uppsala, Sweden) to yield the IgG fraction. ${ }^{14}$

Staining of wax sections. Sections were stained with haematoxylin and eosin or with rabbit antiAcanthamoeba antibody. For the latter procedure sections were first incubated at room temperature with 1:5 normal swine serum (Dakopatts a/s) to block non-specific background staining; they were then incubated in sequence with rabbit anti-Acanthamoeba antibody at dilution 1:10 for 30 minutes, and peroxidase-conjugated swine anti-rabbit immunoglobulin (Dakopatts a/s) at dilution 1:50 for 30 minutes. Phosphate-buffered saline (PBS) was used for all dilutions, and sections were washed with PBS following each of these incubations. 3,3'-Diaminobenzidine (Sigma Chemical Co.) was the chromagen used to identify antibody binding, mixed with $\mathrm{H}_{2} \mathrm{O}_{2}$ in $0.1 \mathrm{M}$ trometamol (Tris) buffer, giving a brown end-product: sections were incubated for three minutes and then washed in tap water for five minutes. Sections were counterstained with haematoxylin.

Culture of corneas for Acanthamoeba. Nonnutrient agar was set in each well of 25-well Petri plates. A lawn of live Escherichia coli was added to the agar surface in each well, and after 8 hours' incubation at $37^{\circ} \mathrm{C}$ the plates were ready for inoculation with tissue. Hemicorneas were homogenised in $150 \mu \mathrm{l}$ of Page's saline with a glass grinder, $10 \mu \mathrm{l}$ of the undiluted homogenate added to each well, and the plate sealed. Corneas
Figure 1D Day 35: paracentral ring-shaped granular opacity and fine superficial peripheral vascularisation (arrow), mydriatic administration. 
from control and infected eyes were cultured and observed by microscopy for seven days. If no growth was seen by seven days, the culture was deemed negative.

\section{Results}

\section{CLINICAL EVALUATION}

The cloudy opacification observed immediately after injection of all corneas cleared completely in control rats before the first examination day 1. Reepithelialisation of the linear incision had also occurred by this time.

All rats inoculated with Acanthamoeba developed keratitis. Certain clinical features were consistently observed. Opacity was granular in character, with corneal thinning superficial to infiltrates apparent in animals with more longstanding keratitis. Minimal, if any, vascularisation and no corneal perforation was seen (Figs 1B, 1C, 1D). No epithelial disease and no signs of anterior uveitis such as nodular infiltrates or hypopyon were observed.

On days 1, 3, and 5 following inoculation grade 3 opacity was observed in almost all rats, maximal at the inoculation site. On day 7 corneal opacity was grade 3 in most rats but had diminished to grade 2 in some. On subsequent examinations a gradual reduction in mean grade of corneal opacity was found (Table I).

\section{EXAMINATION OF PATHOLOGICAL SECTIONS}

Haematoxylin and eosin (H-E) staining of infected corneas identified a marked inflammatory response of neutrophils and macrophages at the inoculation site on day 1 (Fig 2A). A distinction between Acanthamoeba and inflammatory cells was not possible by $\mathrm{H}-\mathrm{E}$ stain but was facilitated by immunoperoxidase staining with anti-Acanthamoeba antibody (Fig 2B). Excystment of many amoebae (Fig 2C) and

Figure 2A Day 1: nonspecific staining by amoebae and inflammatory cells at the inoculation site in an infected cornea. (H-E $\times 540$.) They are very difficult to distinguish.

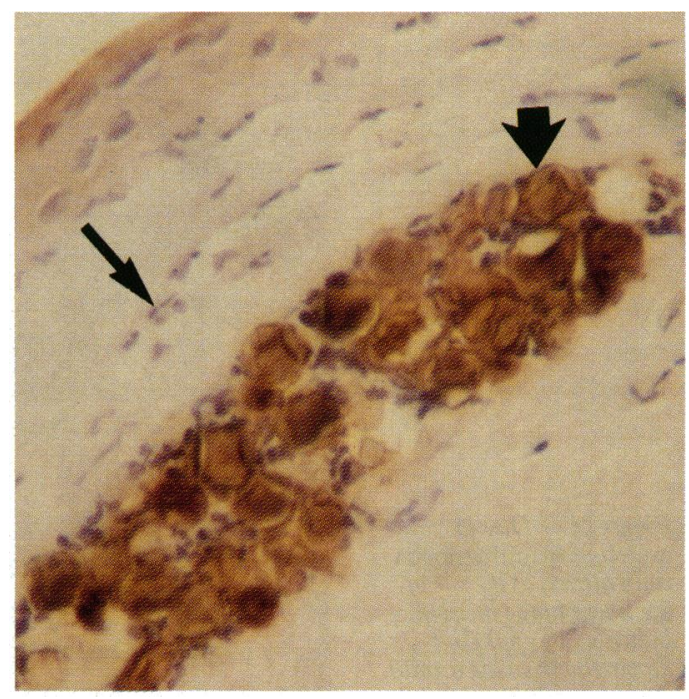

Figure 2B Day 1: Acanthamoeba cysts (large arrow) and migrating polymorphs (small arrow). Little excystment has taken place. (Anti-Acanthamoeba antibody $\times 300$ ).

trophozoite migration from the inoculation site was seen by day 3. A more subdued and mixed inflammatory response was evident by this time, with lymphocytes and monocytes appearing among the neutrophils and macrophages seen on day 1. By day 7 trophozoites were seen throughout the cornea, though large numbers of cysts remained at the inoculation site. Marked stromal thinning was seen overlying the inoculation site in some corneas at day 14 (Fig 2E). Examination of corneas late in disease, from days 21 to 70, consistently revealed amoebae and inflammatory cells to be localised to the inoculation site, surrounded by eosinophilic material.

A gradually diminishing inflammatory response was seen in control corneas at 1,3 and 7 days after mock inoculation (Fig $2 \mathrm{~F}$ ).

\section{CULTURE OF CORNEA}

Positive Acanthamoeba cultures resulted from infected corneas of all animals killed at days 1-49 inclusive. The homogenised corneas of the rat killed at day 70 and all control rats were culturenegative.

\section{Discussion}

The value of an animal model of Acanthamoeba keratitis is attested to by the gravity and increasing prevalence of clinical disease in humans. Study of Acanthamoeba keratitis in an animal model allows control of many of the variable factors involved in human disease. In

Table 1 Mean grade of keratitis observed following injection of Acanthamoeba

\begin{tabular}{lll}
\hline Day & $\begin{array}{l}\text { Number of rats } \\
\text { examined }\end{array}$ & $\begin{array}{l}\text { Mean grade of } \\
\text { keratitis }(S D)\end{array}$ \\
\hline 1 & 24 & $2 \cdot 72(0 \cdot 60)$ \\
3 & 22 & $2 \cdot 76(0 \cdot 61)$ \\
5 & 20 & $2 \cdot 77(0 \cdot 59)$ \\
7 & 18 & $2 \cdot 42(0 \cdot 79)$ \\
10 & 16 & $2 \cdot 08(0 \cdot 81)$ \\
14 & 14 & $1 \cdot 75(0 \cdot 65)$ \\
21 & 12 & $1 \cdot 71(0 \cdot 76)$ \\
28 & 10 & $1 \cdot 93(0 \cdot 83)$ \\
42 & 8 & $1 \cdot 29(0 \cdot 49)$ \\
70 & 6 & $1 \cdot 50(0 \cdot 58)$ \\
\hline
\end{tabular}


Figure 2C Day 1: excysting Acanthamoeba (thin arrow) at the site of the linear blade incision, indicated by epithelial downgrowth (wide arrow). Anti-Acanthamoeba antibody, $\times 300$.)

Figure 2D Day 7: cyst (large arrow) and trophozite (small arrow), each being killed by several inflammatory cells (AntiAcanthamoeba antibody, $\times 760$.)

Figure 2E Day 14: marked thinning of corneal stroma overlying Acanthamoeba cysts which are in deep stroma. These have not migrated and rest on Descemet's membrane. (Anti-Acanthamoeba antibody, $\times 140$.
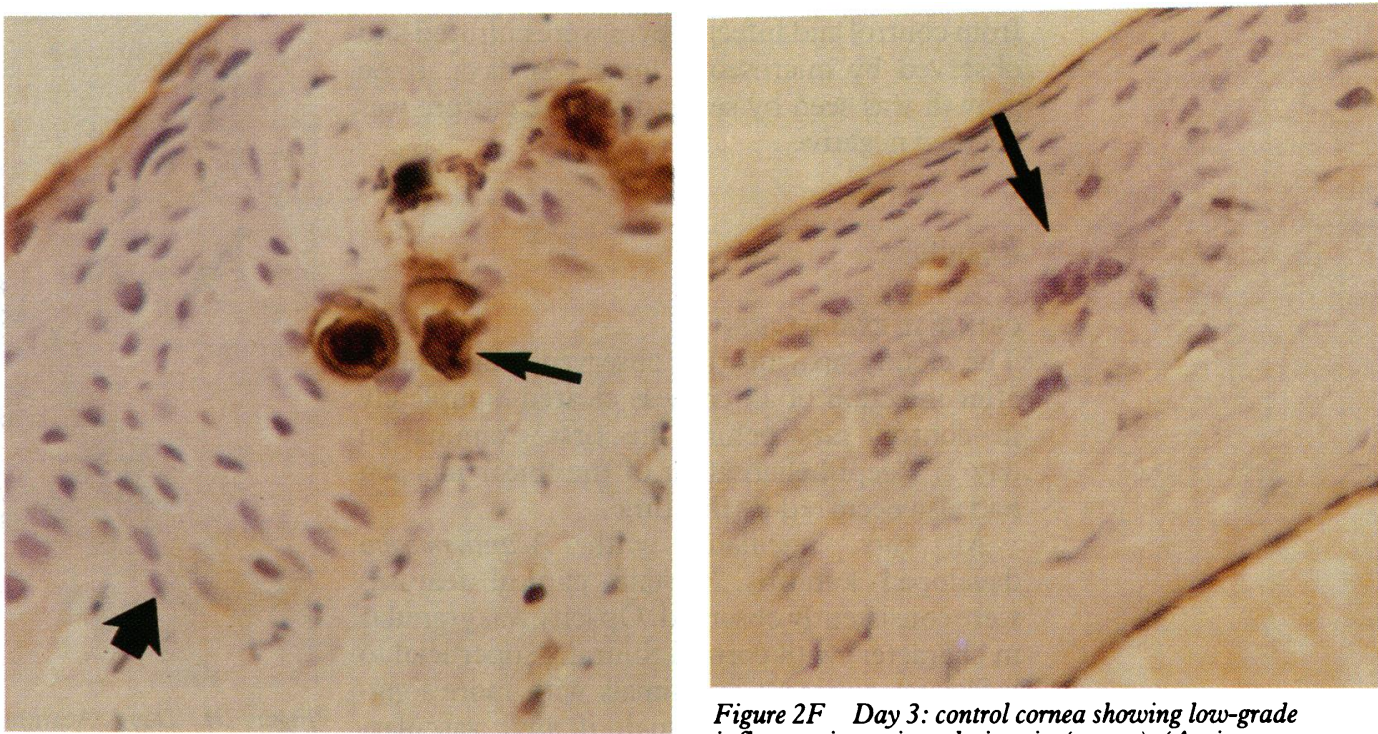

Figure 2F Day 3: control cornea showing low-grade inflammation at inoculation site (arrow). (AntiAcanthamoeba antibody, $\times 190$.)

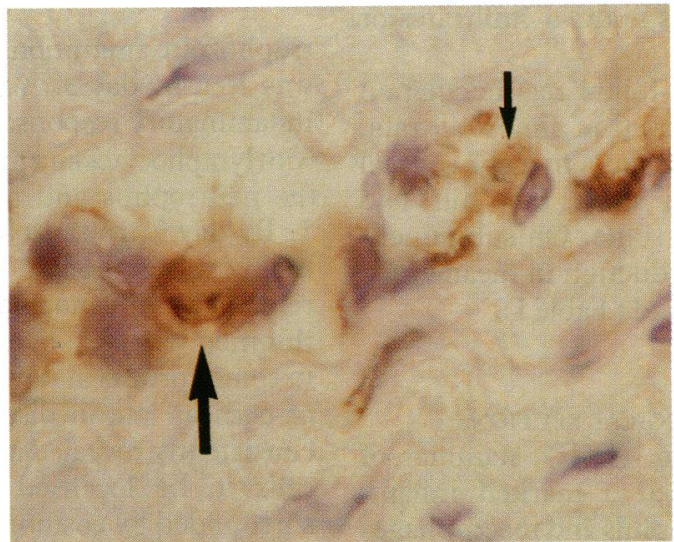

the corneal stroma. Therefore this model does not address questions on the means by which infection arises, but what follows once a large number of cysts reach the stroma. Reproducible keratitis developed in all rat corneas inoculated with $1 \mu \mathrm{l}$ of a suspension of Acanthamoeba at a concentration of $1 \times 10^{6} / \mathrm{ml}$. Because in .clinical practice such an inoculum of cysts would be very unlikely, this may indicate that Acanthamoeba are less infective for the rat cornea than the human. Certain clinical features mimic those observed in human infection, such as minimal corneal vascularisation and diffuse granular opacity formation rather than formation of an abscess. Although acute fulminating infection was not induced, the clinical course of the experimental keratitis is shorter than the human disease, which may remain active for several months. This might be due to differences in rat and human susceptibility to infection. In general, clinical inflammation correlated with histopathological indices of inflammation rather than presence of amoebae as such. In accordance with the report of Font et al. ${ }^{9}$ cysts seemed more resistant to destruction than trophozoites.

Most human specimens on which histopathological descriptions are available originate from corneal transplantation and accordingly describe late stages of the disease, probably modified by drug therapy. Nevertheless, available reports describe changes similar to those described in this report. Disruption and necrosis of stromal lamellae and invasion by amoebae of deep stroma to Descemet's membrane are observed with infiltrates of neutrophils; in some cases there is an added lymphocyte and plasma cell component. ${ }^{65-17}$ However, Acanthamoeba can be seen in tissue relatively free of inflammation. ${ }^{17} 18$

Initial conclusions from this study indicate the clinical features of establis stromal infection may be analysed, pathological study of tissue undertaken, and, in an established model, treatments evaluated. The most appropriate treatment for Acanthamoeba keratitis has yet to be decided: insufficient correlation between in-vitro drug sensitivity tests and clinical response renders it difficult to evaluate new drugs.

In this study Acanthamoeba keratitis was induced by inoculation of organisms directly into that reproducible keratitis of short clinical duration can be induced by intrastromal injection of axenic Acanthamoeba cysts without the need for concomitant immunosuppression by corticosteroid ${ }^{8-10}$ or coinoculation with bacteria. ${ }^{11}$ Thus the rat provides a useful model for investigating the pathology of Acanthamoeba keratitis. Further sequential studies are in progress to 
characterise the cellular composition of the immune response to Acanthamoeba. This model will provide improved understanding of Acanthamoeba-host interactions and ultimately will allow evaluation of specific antimicrobial drugs with and without corticosteroid.

This study was supported in part by grants from Alcon Laboratories Ltd, the British Council for the Prevention of Blindness, and the South-Western Regional Health Authority.

The authors are indebted to Simon Kilvington, BSc, for initially The authors are indebted to Simon Kilvington, BSc, for initially
providing Acanthamoeba cysts and assisting with preparation an providing Acanthamoeba cysts and assisting with preparation an
anti-Acanthamoeba antibody; and to Ian Williams for assistance with the preparation of tissue sections.

1 Puschkarew BM. Über die Verbreitung der Susswasserprotozoen durch die Luft. Arch Protistenk 1913; 28: 323-62. 2 Culbertson CG, Smith JW, Cohen HK, Minner JR. Experimental infection of mice and monkeys by Acanthamoeba. Am f Pathol 1959; 35: 185-97.

3 Martinez A J. Free-Living Amebas: Natural History, Prevention, Diagnosis, Pathology, and Treatment of Disease. Boca Raton: CRC Press, 1985; $127-44$.

4 Jones DB, Visvesvara GS, Robinson NM. Acanthamoeba polyphaga keratitis and Acanthamoeba uveitis associated with 95: 221-32.

5 Nagington J, Watson PG, Playfair TJ, McGill J, Jones BR, Steele ADMcG. Amoebic infection of the eye. Lancet 1974; ii: $1537-40$.

6 Moore MB, McCulley JP, Luckenbach M, et al. Acanthamoeba keratitis associated with soft contact lenses. Am $\mathcal{F}$ Ophthalmol 1985; 100: 396-403.

7 Stehr-Green JK, Bailey TM, Visvesvara GS. The epidemiology of Acanthamoeba keratitis in the United States. Am f Ophthalmol 1989; 107:331-6.

8 Font RI Tapert MJ, Robinson NM, Osato MS, Jones DB. An animal model of Acanthamoeba keratitis. Invest Ophthalmol Vis Sci 1981; 20 (suppl): 8.

9 Font RL, Tapert MJ, Robinson NM, et al. An animal model of Acanthamoeba keratitis. Further studies with emphasis on the early phase destruction of the trophozoites. Invest Ophthalmol Vis Sci 1982; 22 (suppl): 163.

10 Lin J, John T, Sahm D, Rockey JH. Animal model of Acanthamoeba keratitis: effect of corticosteroids. Invest Ophthalmol Vis Sci 1989; 30: (suppl): 37.

11 Badenoch PR, Johnson AM, Christy PE, Coster DJ. Pathogenicity of Acanthamoeba and a corynebacterium in the rat cornea. Arch Ophthalmol 1990; 108: 107-12.

12 Page FC. Taxonomic criteria for limax amoebae, with descriptions of 3 new species of Hartmannella and 3 of Vahlkamphia. tons of 3 new species of Hartmant

13 Warhurst DC. Naegleria and Acanthamoeba in tissue sections. In: Edwards JMB, Taylor CED, Tomlinson AH, eds. Immunofluorescence techniques in diagnostic microbiology. London: HMSO, 1982; 46-8.

14 Tucker DF, Begent RHJ, Hogg NM. Characterization of immune complexes in serum by adsorption on staphylococcal protein A: model studies and application to sera of rats bearing a Gross virus-induced lymphoma. 7 Immunol 1978; 121: $1644-51$.

15 Lund OE, Stefani FH, Dechant W. Amoebic keratitis: a clinicopathological case report. $B r f$ Ophthalmol 1978; 62 : 373-5.

16 Auran JD, Starr MB, Jakobiec FA, Acanthamoeba keratitis. A review of the literature. Cornea 1987; 6: 2-26.

17 Garner A. Pathology of corneal acanthamoebic infection. In: Cavanagh HD, ed. The cornea: transactions of the World Congress on the Cornea III. New York: Raven Press, 1988: 535-9.

18 Blackman HJ, Rao NA, Lemp MA, Visvesvara GS. Acanthamoeba keratitis successfully treated with penetrating keratoplasty: suggested immunogenic mechanisms of action. Comea 1984: 3: 125-30. 